\title{
Claude Didry, L'Institution du salariat : droit et salariat dans l'histoire
}

Paris, La Dispute, coll. « Travail et salariat », 2016

\section{Thierry Kirat}

\section{(2) OpenEdition}

\section{Journals}

Édition électronique

URL : http://journals.openedition.org/travailemploi/7416

DOI : $10.4000 /$ travailemploi.7416

ISSN : 1775-416X

Éditeur

DARES - Ministère du Travail

Édition imprimée

Date de publication : 1 janvier 2017

Pagination : 106-108

ISSN : 0224-4365

Référence électronique

Thierry Kirat, "Claude Didry, L'Institution du salariat: droit et salariat dans I'histoire », Travail et Emploi [En ligne], 149 | janvier-mars 2017, mis en ligne le 11 juillet 2019, consulté le 24 septembre 2020. URL :

http://journals.openedition.org/travailemploi/7416 ; DOI : https://doi.org/10.4000/travailemploi.7416 


\section{L'Institution du salariat : droit et salariat dans l'histoire}

\section{Claude Didry}

Paris, La Dispute, coll. « Travail et salariat », 2016, 244 p.

\section{Lu par Thierry Kirat"}

Ce livre passionnant donne à voir une belle enquête socio-juridique et sociohistorique sur la construction du contrat de travail, de la classification des emplois, de la branche et de l'entreprise. Autour du fil directeur qu'est le droit, sa forte originalité est d'articuler toutes ces dimensions du monde du travail. Parcourant avec une grande attention deux siècles d'histoire sociale, l'auteur organise l'argumentation en sept chapitres thématiques qui n'en suivent pas moins un ordre chronologique permettant de saisir l'épaisseur du temps - temps du louage d'ouvrage puis du marchandage, enfin temps des questions et des propositions sur l'horizon du travail.

Les trois premiers chapitres constituent le socle de l'ouvrage. La manière dont ils s'enchaînent montre ce qu'a eu de révolutionnaire l'institution du contrat de travail par le premier Code du travail en 1910. Mettant en évidence les « règles du jeu posées par la Révolution et l'Empire » (p. 19), le chapitre 1 invite à un dépaysement intellectuel en expliquant que l'activité de travail dans le monde ouvrier s'organise alors autour du concept de « louage d'ouvrage » défini en 1804 par le Code civil comme un « "contrat par lequel l'une des parties s'engage à faire quelque chose pour l'autre moyennant un prix convenu entre elles", contrat précisé dans une vingtaine d'articles (1779 à 1799) ( pp. 18-19). Claude Didry soutient que le nouvel ordre juridique post-révolutionnaire intègre le monde ouvrier dans le Code civil, et plus précisément dans le « louage d'ouvrage » par « devis et marchés » : il s'agit d'une opération par laquelle un « donneur d' ordre » confie à autrui la réalisation d'un ouvrage, sur la base d'un devis. La fin des corporations a signifié l'entrée dans le droit civil, et la mise en place de régulations collectives, notamment par les premiers Conseils de prud'hommes qui, à partir de 1806, deviennent des institutions de régulation du prix des ouvrages. Dans le monde du louage d'ouvrage, la production est commandée par un commerçant, un négociant, qui fournit la matière et en confie l'exécution à un « ouvrier », qui luimême délègue des parties du procès de production. L'organisation du travail et de la production dans la soierie lyonnaise avec les canuts en est un cas topique. Les premiers Conseils de prud'hommes assurent une régulation collective de ce système complexe

\footnotetext{
* Institut de recherche interdisciplinaire en sciences sociales (Irisso), université Paris Dauphine.
} 
qui met en relation « négociants-fabricants », chefs d'atelier, contremaîtres, apprentis, etc. Là où cette organisation butte, c'est sur les prix : les tarifs. La concurrence entre preneurs d'ouvrage laisse émerger une « entr'exploitation » ouvrière, une réduction des coûts par des prestations sous-payées ou par le sweating-system du travail familial, dès lors que la faiblesse des prix contraint les ouvriers à mettre leur famille, y compris les enfants, à contribution. Ce genre de « marchandage » est au cœur du chapitre 2, qui présente clairement les processus, politiques et sociaux, qui ont conduit au décret du 2 mars 1848 abolissant le marchandage et à l'arrêt de la Chambre sociale de la Cour de cassation qui, en 1901, en a limité la portée pour n'interdire le marchandage que lorsque celui-ci porte préjudice à l'ouvrier.

$\mathrm{Si}$, comme l'écrit l'auteur, « le salariat ne peut plus [...] être considéré comme un donné que le capitalisme trouve sur ses fonts baptismaux » (p. 66), il faut scruter le passage du louage d'ouvrage au contrat de travail. Le chapitre 3 s'y attelle, en relatant entre autres les débats politiques, législatifs et juridiques de la Belle Époque, sur les moyens de donner un cadre nouveau au travail. C'est au sein de la Société d'études législatives qu'émerge, sous la plume de Camille Perreau ${ }^{1}$, une rupture avec les catégories du Code civil : « Il convenait d'assurer une situation juridique identique à des catégories de personnes qui, sous des noms parfois différents, n'ont en somme que la même situation économique » (p. 77). Claude Didry présente avec finesse les termes du débat d'alors sur le critère du contrat de travail, entre dépendance économique et subordination. Il prolonge son étude sur la réforme des conseils de prud'hommes, la durée du travail, la protection sociale, le délai-congé. On voit, en passant, les midinettes ${ }^{2}$ entrer dans le salariat. Mais, surtout, les innovations juridiques et institutionnelles transforment le sens même du travail vers plus d'individuation ; les travailleurs émergent, à la place des ouvriers « relevant de tel ou tel métier » (p. 104).

Symboliquement, entrer dans le contrat de travail, c'est entrer dans la manufacture concentrée $^{3}$, c'est aussi entrer dans un système de classification, dont une part de la logique découle de la pratique syndicale et de l'innovation institutionnelle qu'a représentées la convention collective de branche. Le chapitre 4 analyse avec minutie cette « grande classification », dont le pivot est alors l'établissement, niveau où s'inscrit une « hiérarchie salariale », « lieu de rattachement des contrats de travail » (p. 109). Le chapitre 5 soutient que, depuis la politique industrielle des « champions nationaux », le rôle-pivot de l'établissement s'estompe, voire cède la place à l'entreprise. Les mutations industrielles et les suppressions d'emploi mettent en jeu une grammaire du travail devenu objet d'un marché, source de coûts, qu'il convient alors d'ajuster.

1. Professeur d'économie politique à la faculté de droit de Paris, Camille Perreau fut rapporteur de la question de la définition du contrat de travail au sein de la Société d'études législatives. Député des Bouches-du-Rhône, inscrit au groupe radical indépendant, de 1898 à 1902, il participa aux travaux de la Commission du travail de la Chambre des députés sur le contrat de travail et le Code civil.

2. Nom donné aux couturières à domicile travaillant principalement pour de grands magasins.

3. Par opposition aux ateliers familiaux, dispersés, la manufacture concentrée regroupe dans un même lieu outillage et travailleurs dont le nombre peut aller jusqu'à plusieurs centaines. 
La contrepartie de ces transformations industrielles et sociales a été trouvée dans la mise en place de filets de protection sociale. Le chapitre 6 approfondit l'analyse des restructurations et met l'accent sur ce qui accompagne l'individualisation du contrat de travail : une conflictualité diffuse, qui va des conditions de travail à la place des comités d'entreprise dans la mise en discussion des restructurations. Mais Claude Didry va plus loin : il met en quelque sorte en abîme des entreprises dont les frontières sont de plus en plus floues, avec la quête des salariés et des syndicats de faire advenir des unités économiques et sociales. À cet égard, l'auteur insiste sur le cas des maisons de Champagne appartenant au groupe LVMH où, à l'occasion de la contestation du plan social proposé par la direction, le tribunal de Châlons-en-Champagne a, en 1993, opéré « une redéfinition du périmètre des maisons champenoises d'Épernay regroupées [...] dans une même unité économique et sociale [...] autour de Moët-et-Chandon » (p. 187).

Finalement, l'ultime chapitre propose quelques orientations de débats et de réflexions sur l'horizon du travail, à partir de l'histoire du salariat et de ses enjeux telle qu'elle a été dressée tout au long de l'ouvrage. Claude Didry y explore le principe d'une sécurité sociale professionnelle, qui suppose selon lui que, d'une manière ou d'une autre, les acteurs retrouvent, au-delà de l'entreprise, « le travail et l'industrie » (p. 238).

Il s'agit en somme d'un ouvrage à lire car il fait réfléchir, en particulier sur les enjeux des représentations juridiques du monde du travail. Celles-ci sont en effet plus puissantes que les lectures économiques de la législation de protection de l'emploi ne le laissent penser : elles instituent les acteurs et donnent les références des actions individuelles et collectives. Le fait que le dernier chapitre revienne sur l'idée du droit social de Georges Gurvitch n'est certainement pas un hasard. L'auteur de cette note de lecture recommande en particulier les trois premiers chapitres, qui constituent une belle enquête du passage du contrat de louage d'ouvrage au contrat de travail. Elle aurait d'ailleurs gagné à être mise en rapport de manière plus systématique avec les formes d'organisation de la production, de la manufacture dispersée à la manufacture concentrée. 